\title{
A Simple Non-Invasive Biomarker Can Reflect Both the Acute and Chronic Pulmonary Impact of Patent Ductus Arteriosus (PDA) Shunting
}

\author{
Alona Bin-Nun ${ }^{1}$, Irina Shchors ${ }^{2}$, Rawan Abu-Omar², Yair Kasirer², Francis Mimouni², and \\ Cathy Hammerman ${ }^{1}$ \\ ${ }^{1}$ Shaare Zedek Med Ctr \\ ${ }^{2}$ Shaare Zedek Medical Center
}

January 3, 2022

\begin{abstract}
The SFR (Sp02/Fi02 ratio) offers a continuous, non-invasive reflection of pulmonary function regardless of whether the baby is ventilated or breathing spontaneously. We hypothesized that significant PDA shunting would impair pulmonary oxygen diffusion, in turn, reflected by decreased SFR; and that early PDA related decreases in SFR will predict subsequent chronic lung disease (CLD). Methods: We retrospectively examined records from preterm neonates $<30$ weeks gestational age. Ductal shunting was graded for severity by first week echocardiogram. SFR was calculated as SpO2/Fi02 and recorded on day 7 of life and at 36 weeks postmenstrual age (PMA). Results: We studied 104 infants: 65 with closed duct; 17 with hemodynamically insignificant PDA and 22 with hemodynamically significant (hsPDAs). CLD developed in 9 (14\%) of those with closed ducts; $6(35 \%)$ of those with hisPDA; and in $12(55 \%)$ of those with hsPDA $(\mathrm{p}=0.005)$. Babies with hsPDA had significantly lower SFR values at both time points. SFRs in babies with hisPDA were decreased at 1 week postnatally, but were similar to those of babies with closed ducts at 36 weeks. SFR at 36 wks. was decreased only in infants with hsPDA [[467[461,467] vs. $467[413,471]$ vs. $369[262,436]$ respectively; $p=0.000148]$. Using ROC curve analysis, week 1 SFR was strongly associated with hsPDA (AUC $=0.770 ; \mathrm{p}<0.0001)$ and highly predictive $(\mathrm{AUC}=0.801 ; \mathrm{p}<0.0001)$ of $\mathrm{CLD}$ at 36 weeks PMA. Conclusion: Early decreases in SFR reflect both the acute and chronic pulmonary impact of PDA shunting, possibly providing the missing link supporting an association between hemodynamically significant PDA and subsequent CLD.
\end{abstract}

\section{Hosted file}

SFR 2712.docx available at https://authorea.com/users/453654/articles/551437-a-simple-noninvasive-biomarker-can-reflect-both-the-acute-and-chronic-pulmonary-impact-of-patentductus-arteriosus-pda-shunting 
Figure 1.
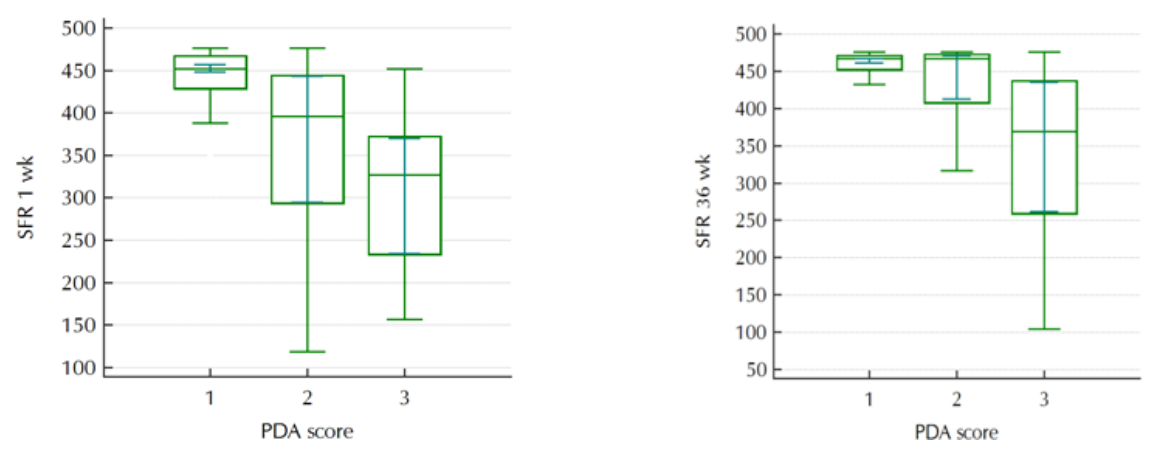

SFR 1 wk

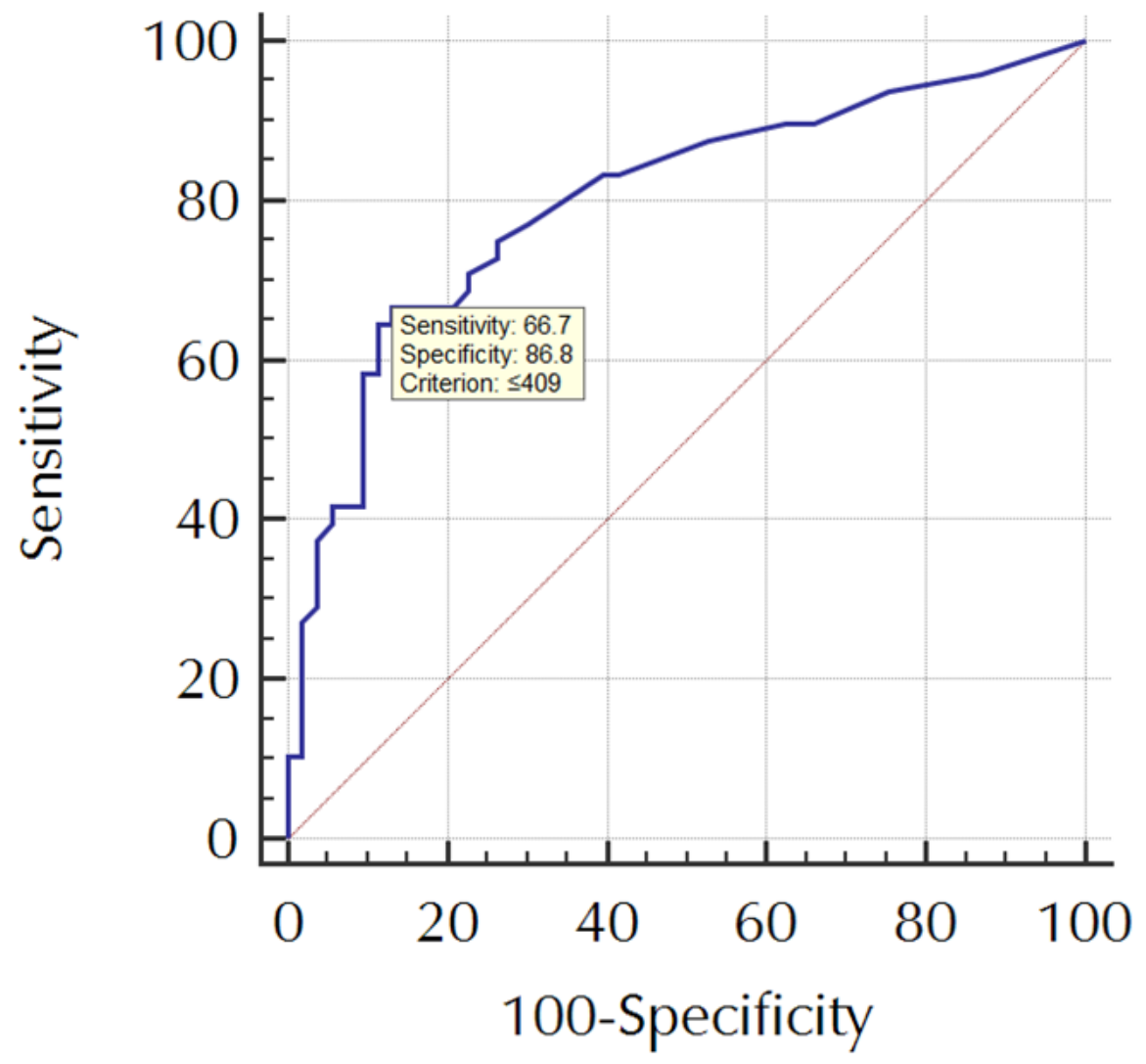

